\title{
Updates on Percutaneous Radiologic Gastrostomy/Gastrojejunostomy and Jejunostomy
}

\author{
Ji Hoon Shin* and Auh-Whan Park ${ }^{\dagger}$ \\ *Department of Radiology and Research Institute of Radiology, Asan Medical Center, University of Ulsan College of Medicine, Seoul, Korea, \\ ${ }^{\dagger}$ Department of Radiology, University of Virginia, Charlottesville, VA, USA
}

Gastrostomy placement for nutritional support for patients with inadequate oral intake has been attempted using surgical, endoscopic, and, more recently, percutaneous radiologically guided methods. Surgical gastrostomy has been superseded by both endoscopic and radiologic gastrostomy. We describe herein the indications, contraindications, patient preparations, techniques, complications, and aftercare with regard to radiologic gastrostomy. In addition, we discuss the available tube types and their perceived advantages. There remain some controversies regarding gastropexy performance and primary percutaneous gastrojejunostomy. Percutaneous jejunostomy is indicated for patients whose stomach is inaccessible for gastrostomy placement or for those who have had a previous gastrectomy. (Gut Liver 2010;4(Suppl. 1): S25-31)

Key Words: Gastrostomy; Gastrojejunostomy; Jejunostomy; Enteral feeding; Interventional procedures

\section{INTRODUCTION}

Percutaneous radiologic gastrostomy (PRG), also known as "radiologically inserted gastrostomy," is the traditional, nonendoscopic procedure. ${ }^{1}$ The tube is inserted directly through the abdominal wall into the stomach. Tract dilatation and tube insertion through the abdominal wall carry a risk of displacing the stomach and of placing the tube in the peritoneal cavity. This is prevented by placing "gastropexy" sutures. The main indication is dysphagia resulting from upper gastrointestinal cancer because PRG avoids the use of a peroral tube that could disseminate tumor cells into the stoma. ${ }^{2}$

Percutaneous endoscopic gastrostomy (PEG) is the most readily available technique. The robust retention bumpers are particularly useful in stroke patients who require long-term feeding and who might attempt to pull the tube out as a result of cognitive function. ${ }^{1}$ The success of PEG is reduced by anatomic abnormalities (e.g., high lying stomach, surgically altered stomach), obesity, and difficult passage of endoscopy (e.g., esophageal strictures). Neither the position of the liver nor that of colon is identified. Two types of pull-gastrostomy and push-gastrostomy are used.

Percutaneous radiologic gastrojejunostomy (PRGJ) is a procedure that involves placing a tube tip into the jejunum through a puncture of the stomach under fluoroscopic guidance. ${ }^{3}$

Percutaneous radiologic jejunostomy (PRJ) is indicated for patients with a history of chronic aspiration, gastric surgery (i.e., gastrectomy), or abnormal gastric position.

The aims of this article are to describe techniques and outcomes of PRG, PRGJ and PRJ.

\section{PERCUTANEOUS RADIOLOGIC GASTORSTOMY (PRG)}

\section{Indication/Contraindication}

PRG is most commonly indicated for nutritional support for patients with disorders of swallowing due to stroke and other neurologic disorders, esophageal obstruction, and lesions of the head, neck, and mediastinum (e.g., recent surgery and radiation therapy). Diversion of feedings from esophageal leaks caused by recent surgery

\footnotetext{
Correspondence to: Ji Hoon Shin

Department of Radiology, Asan Medical Center, University of Ulsan College of Medicine, 86 Asanbyeongwon-gil, Songpa-gu, Seoul 138-736, Korea

Tel: +82-2-3010-4380, Fax: +82-2-476-0090, E-mail: jhshin@amc.seoul.kr DOI: $10.5009 / \mathrm{gnl} .2010 .4 . S 1 . S 25$
} 
or trauma and diversion of gastroenteric contents in cases of gastric outlet or proximal small bowel obstruction and gastroparesis (e.g., diabetic gastropathy) are also indicated.

With recent innovations in placement technique and gastrostomy tube, there are now fewer contraindications. PRG is contraindicated in patients with uncorrectable coagulopathy and unsatisfactory percutaneous access to the stomach (e.g., massive hepatosplenomegaly, interposed colon). The presence of varices as in portal hypertension significantly increases the risk of bleeding, and gastrostomy is best avoided. ${ }^{4}$ Surgically altered stomachs (e.g., subtotal gastrectomy) could be challenging, however, understanding the patient's anatomy makes PRG easier. For massive ascites, preprocedural paracentesis and/or gastropexy (to prevent peritubal leakage) is necessary. For interposed colon, infracolic access under CT or US guidance is recommended. Ventriculoperitoneal shunt was controversial as contraindications, however, recently it is not regarded as contraindications because percutaneous gastrostomy did not increase infection risk. ${ }^{5}$

\section{Patient preparation}

Coagulation study should be checked and preliminary CT or US examination can be used to rule out overlying viscera such as left hepatic lobe or transverse colon. Alternately, fluoroscopic delineation of the colon is made by giving $100-200 \mathrm{~mL}$ of barium sulfate the night before the procedure. This can be administered through a nasogastric tube, which is placed bedside the evening before the procedure to empty the stomach as well as to inflate the stomach during the procedure. If there is a difficulty in placing the nasogastric tube, an angiographic catheter (e.g., a 5 Fr Cobra catheter) is passed into the stomach

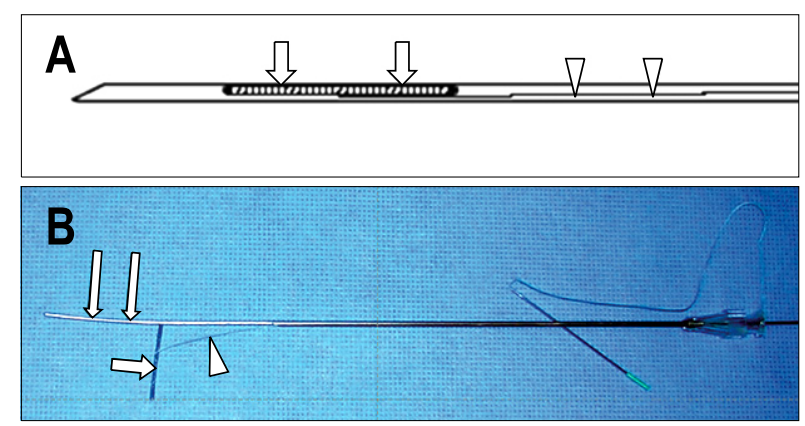

Fig. 1. A Cope suture anchor. (A) The anchor, which is made of a short metal bar (arrows) attached to a surgical suture (arrowheads), is preloaded into a 17-gauge puncture needle. (B) The anchor (arrow) is deployed when a guide wire (long arrows) is advanced through the puncture needle. The distal end of the suture string (arrowhead) is attached to the suture needle for fixation to the skin. with the aid of a hydrophilic guide wire (GW) under fluoroscopic guidance immediately prior to the procedure. This is entirely atraumatic and is nearly always successful.

Sedation is started with $1 \mathrm{mg}$ midazolam and 25 micrograms fentanyl with further increments as needed. Conscious sedation should be given judiciously in patients with head and neck cancers or respiratory compromise. Continuous blood pressure and pulse oximetry monitoring should be performed throughout the procedure.

The key to success is adequate gastric distension in combination with a smooth-muscle relaxant (e.g., $20 \mathrm{mg}$ hyoscine butylbromide). The stomach is inflated with $600-1,000 \mathrm{~mL}$ of room air. This extends the stomach below the costal margin and the liver and displaces the transverse colon from the epigastrium, thereby facilitating stomach puncture.

\section{Technique}

Gastric fixation is only required for tubes that are pushed through the abdominal wall (i.e., in PRG, PRGJ). There are two kinds of anchor devices made of short metal bars attached to a surgical suture - Cope suture anchor (Cook, Bloomington, IN, USA) (Fig. 1) and T-fastener (Boston Scientific, Natick, MA, USA). Anchors are inserted into the stomach using gastropexy set. Tract dilatation with fascial dilators exerts a large amount of force, and multiple (up to four) anchors are required for $12 \mathrm{Fr}$ tubes or larger (Fig. 2). Although the performance of gas-

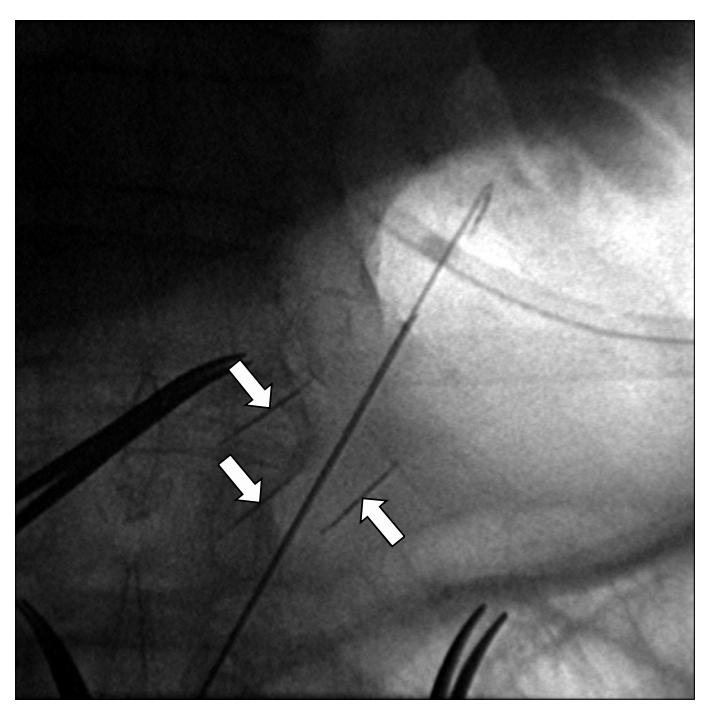

Fig. 2. Percutaneous radiologic gastrostomy with multiple gastropexy. Three sets of anchors (arrows) are inserted into the stomach at the corners of a triangle. The puncture is done at the center of the triangle and a 14-Fr pigtail-retained tube is inserted successfully (not shown). 
tropexy has remained somewhat controversial, one prospective randomized study has shown that it reduces the

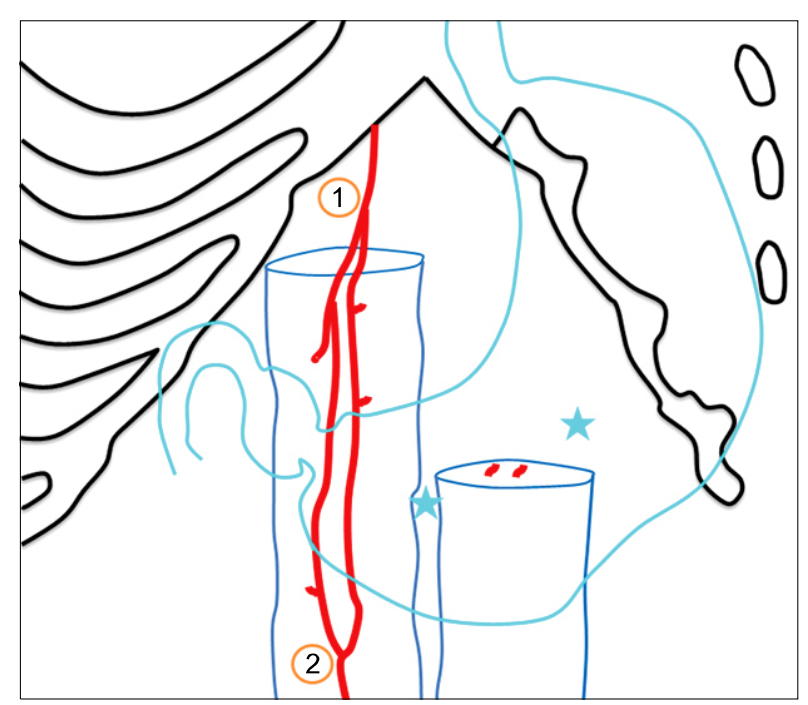

Fig. 3. Gastric puncture (asterisks) is performed at the mid to distal gastric body, equidistant from the greater and lesser curvatures. It should be lateral to the rectus muscle or in the midline to avoid puncture of epigastric arteries ( 1 and 2 indicate the superior and inferior epigastric arteries, respectively). risk of initial intraperitoneal tube placement and obviates subsequent tube migration through the formation of adhesions. ${ }^{6}$ For PRGJ or PRJ tube placement, gastropexy is standard.

If gastropexy is not used, continuous air insufflations may be necessary during the procedure to keep the stomach distended. In patients with partial gastrectomy, the remnant may be located high under the costal margin, requiring complex angulation and a longer access needle.

Mid to distal body of the stomach, equidistant from the greater and lesser curvatures, is the best site for puncture to prevent arterial injury (Figs. 2 and 3). In addition, puncture should be made lateral to the rectus muscle or in the mid-abdomen to avoid injury of superior or inferior epigastric artery (Fig. 3).

Gastric puncture is performed with a 17-gauge puncture needle preloaded with an anchor (Cope gastrointestinal suture anchor sets; Cook). An intragastric position is confirmed by aspiration of air into a syringe and/or injection of contrast, outlining gastric rugal folds. The anchor is then deployed by passing a GW through the needle (Fig. 4). The GW and needle are subsequently removed and the stomach is gently approximated to the anterior abdominal wall by gentle traction on the anchor. In cases of
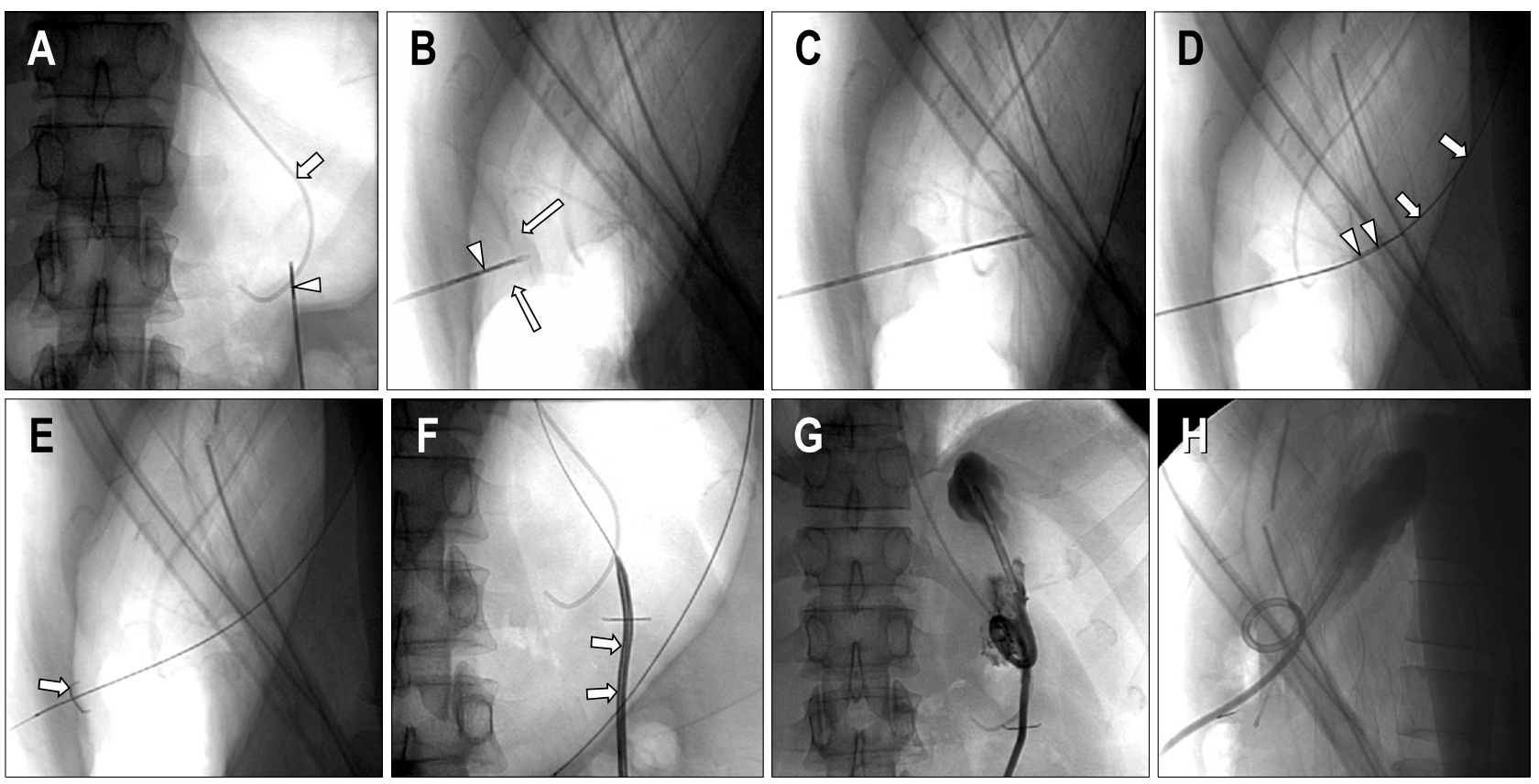

Fig. 4. Percutaneous radiologic gastrostomy with a one-anchor technique (courtesy by K.H. Lee, M.D.). (A, B) The stomach is inflated with a vascular catheter (arrow) and a puncture needle preloaded with an anchor (arrowheads) is advanced under frontal and lateral fluoroscopic guidance. Gastric tenting (long arrows) made by the anterior stomach wall is clearly seen in a lateral view. (C) With a brief thrust, the anterior stomach wall is punctured. (D) With advancement of a guide wire (arrows), the anchor (arrowheads) is deployed within the stomach. (E) By traction of the anchor (arrow), the anterior stomach wall is apposed to the abdominal wall. (F) The puncture tract is dilated serially with dilators (arrows). (G, H) A 14-Fr pigtail-retained tube is placed and its intragastric location is confirmed with contrast-agent injection in frontal and lateral views. 
four anchors, each anchor is inserted into the stomach at the corners of the $2-\mathrm{cm}$ square, and the center of the anchor square is punctured. The puncture should be made with a brief, deliberate thrust so as not to push the anterior gastric wall away from the anterior abdominal wall. Usually, the puncture needle is directed vertically down or slightly toward the fundus. However, if conversion to a PRGJ tube is anticipated, the needle should be directed toward the pylorus. Fascial dilators, of adequate diameter to accommodate the feeding tube, are introduced over the 0.038-inch GW to dilate a tract. Because of the thick muscular wall of the stomach, the dilator can easily push the stomach wall forward rather than dilating the puncture hole within the wall. If this happens, the GW may prolapse outside the stomach into the peritoneum. A GW with the proper stiffness and attention during manipulation helps avoid this problem.

Then, selected PRG tube is inserted over a GW. Following tube placement, contrast is injected and frontal and lateral views of the upper abdomen are obtained to confirm proper position (Fig. 4). The feeding tube is secured to the skin with sutures or commercially available retaining devices.

If difficulties with fluoroscopic guidance are anticipated, a PRG tube may be placed under CT or US guidance.

\section{Tube choice}

\section{1) Pigtail-retained (loop-retained) tubes}

They are small- bore tubes with 10-14 Fr diameter available (Wills- Oglesby percutaneous gastrostomy catheter, Cook). They (especially, $10 \mathrm{Fr}$ ) are easy to be occluded, and tube displacement is a continual problem, requiring regular maintenance and exchange. A $12 \mathrm{Fr}$ or larger pigtail-retained gastrostomy tube is usually used. To avoid the risk of duodenal perforation, these tubes are preferentially oriented toward the gastric fundus. They can be placed fast and safe without full sedation, so, advantageous for much debilitated patients.

\section{2) Balloon-retained tubes}

They are large-bore tubes with 14-16 Fr diameters and more secure than pigtail-retained tubes. They are intended to be used as replacement tubes for a mature tract. Balloon can deflate, leading to tube dislodgement. Higher diameter profile is inevitable because valve is outside the body.

\section{3) Bumper-retained (mushroom-retained) tubes}

They are very large-bore ( $>20 \mathrm{Fr}$ ) tubes and have traditionally been used only for PEG. They are very secure because their fixed retention mechanism cannot be unlocked or deflated. ${ }^{7}$ They are very durable, so, advantageous for the patients who need long-term use with less maintenance/exchange. However, because the tube must pass through the mouth, this type of gastrostomy may be predisposed to a higher rate of infection than conventional PRG. Collapsible bumpers allow removal by external traction, avoiding the need for further endoscopy and sedation. ${ }^{1}$ However, silicone that is bathed in gastric acid hardens over a period of several months and especially tubes with a smaller stoma (e.g., 14 Fr) may become impossible to remove by traction.

\section{4) Low-profile tubes}

They are button gastrostomy tubes and have a small hub containing a valve at skin level. They are less prone to occlusion. There are two main groups of gastrostomy buttons- mushroom tip and retention balloon. They require a feeding extension, but are preferable for children and for active patients because they are easily hidden under the patient's clothing. ${ }^{1}$ Originally designed as replacement devices, they are easily inserted at the primary procedure using a PRG technique with gastropexy. ${ }^{8}$ Accurate abdominal-wall tract length measurement is important when placing button because the device has a finite shaft length. Tract length is measured using a standard angioplasty balloon catheter inflated within the gastric lumen. ${ }^{8}$

\section{Modification of PG placement}

\section{1) Pull-type gastrostomy tube placement}

This is a hybrid of PRG and PEG, and is also called "peroral image-guided gastrostomy" or "radiologic peroral gastrostomy." Following successful percutaneous gastric access under fluoroscopic guidance, a 5 to $7 \mathrm{Fr}$ angiographic catheter and GW are used to cross the gastroesophageal junction to the esophagus. The catheter and GW are advanced in a retrograde direction and pulled out of the patient's mouth. After exchanging the GW for a "traction wire" or preferably an exchange-length GW, a pull-type gastrostomy tube is introduced coaxially, passing in an antegrade direction into the esophagus and out the anterior abdominal wall. The tapered tip of the gastrostomy tube is then cut to the desired length. Larger-bore tubes, similar to those passed endoscopically, can be used. A 20 Fr mushroom-retained tube (removable pull-PEG, Medical Innovations, Draper, UT, USA) is commonly used.

\section{2) One-anchor technique (Fig. 4)}

Controversy also exists as to the number of anchors 
that is sufficient. With one-anchor technique, a single anchor is inserted through the preloaded 17-gauge puncture needle. After confirming its location within the stomach, the anchor is released by pushing with the GW. The anchor is pulled firmly toward the anterior abdominal wall; serial dilators are introduced over the GW through the same tract. Finally, a gastrostomy tube (10 Fr to $16 \mathrm{Fr}$ ) is introduced over the GW into the stomach. ${ }^{9}$ Although the one-anchor technique is a simple procedure, risk of anchor dislodgment and related peritonitis could be increased.

\section{Outcomes}

Technical success of PRG placement approaches $100 \%$. PRG has a slightly higher success rate compared with that of PEG and is often successful when the PEG technique fails. The main reason for failure is a stomach positioned in the chest, which precludes puncture. ${ }^{1}$

30-day mortality from all causes has been reported to be from around $4 \%^{10}$ to $8 \%^{11}$ depending on the severity of the underlying illness, according to one meta-analysis of the literature. ${ }^{11}$

\section{Complications}

Major complications (peritonitis, bleeding, deep stomal infection, aspiration, displacement of the tube requiring a repeat procedure, and sepsis) are under $8 \% .{ }^{11}$ Minor complications such as superficial stomal infection, minor peritubal leakage, or tube dislodgement are reported in $5-10 \%$ of cases. ${ }^{11}$

Peritonitis associated with PRG is a rare but most serious major complication. This complication is caused by either from intraperitoneal leakage around a puncture site or from tube migration and erosion causing frank gastric perforation. Upright plain radiograph demonstrating in- creasing pneumoperitoneum is a reliable sign of intraperitoneal leakage. Tube feeding should be held, and surgical consultation (e.g., exploratory laparotomy) should be considered, if necessary.

Gastric bleeding after PRG and PEG has been reported to occur at a frequency of $1-3 \% .^{12}$ Puncture of the stomach with beveled needles whose tips afford extensive cutting action can be associated with vascular injury. ${ }^{13}$ Transarterial embolotherapy is useful for controlling massive hemorrhage (Fig. 5). ${ }^{9}$

Deep stomal infection is more frequent in PEG than in PRG because tube is passed through the mouth/oropharynx and can be contaminated by oral flora, resulting in wound infection. Prophylactic antibiotics is needed in PEG, however, prophylactic antibiotics is not a routine practice for PRG. Appropriate antibiotics after the development of wound infection is usually satisfactory.

Aspiration pneumonia occurs less frequently with PRG than with PEG because of profound sedation and the technique used during the endoscopic procedure in PEG. For patients with frequent gastroesophageal reflux (GER), primary or conversion PRGJ, or PRJ could be alternatives.

Dislodged tubes are repositioned or replaced with the same catheter or a balloon replacement tube. A partially closed tract can usually be salvaged with a hydrophilic GW and a 5 Fr catheter and be redilated. ${ }^{1}$

Buried bumper syndrome is usually a complication with low profile button or balloon tubes. Excessive traction, caused either by the tube being fitted too tightly or by manipulation, can cause the internal bumper to migrate into the gastrostomy tract and become overgrown by gastric mucosa. In the case of a deeply buried rigid retention disk, the options are limited. These either have to be removed surgically or have to be left and a new gastrostomy inserted at a separate site. ${ }^{1}$

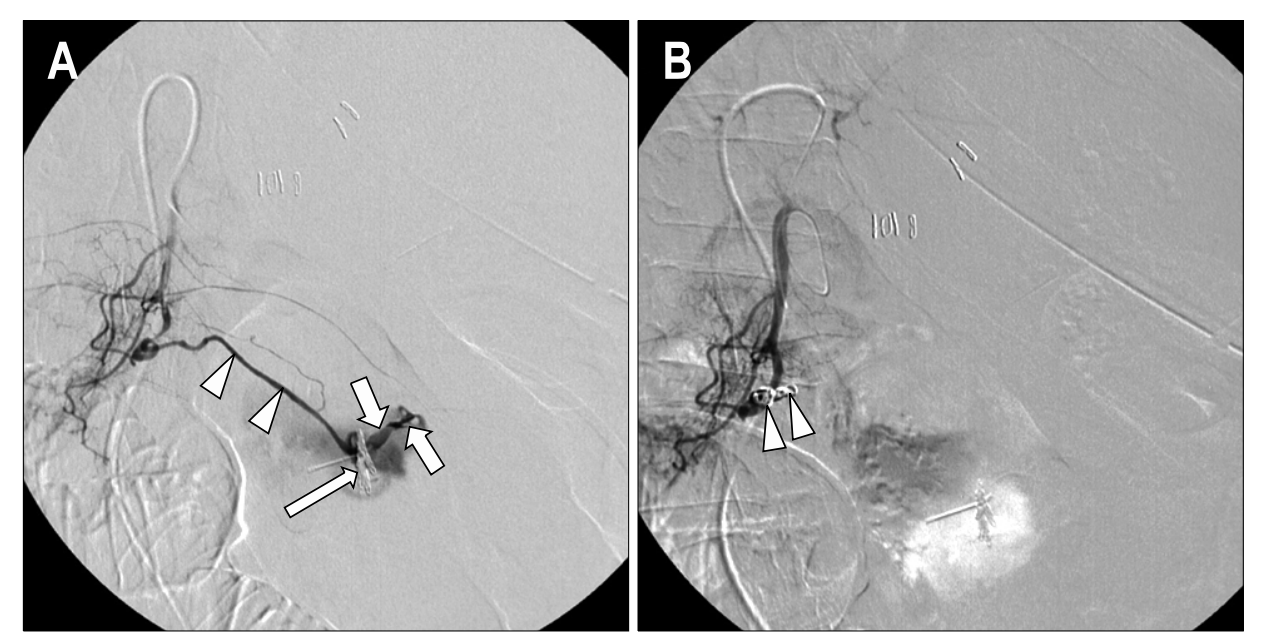

Fig. 5. Embolotherapy for massive bleeding after percutaneous radiologic gastrostomy. (A, B) Bleeding (contrast-agent extravasation, arrows) persisted from the right gastroepiploic artery (arrowheads in A) even after attempts at endoscopic hemostatic with clips (long arrows). Embolotherapy was performed successfully with Gelfoam particles and coils (arrowheads in B). 


\section{Postprocedural and follow-up care}

Vital signs and serial abdominal examination must be closely followed, looking for signs of peritonitis that may indicate leakage of gastric contents. Pneumoperitoneum on routine imaging is not unexpected and slowly resolves over 1-3 days.

After the procedure, the stoma is kept dry, with intermittent antiseptic cleaning. The tube should remain clamped for the first 24 hours following placement, before attempting feedings. If overnight output is not excessive, and if the abdominal examination is benign, feedings can be started the next morning. If the tube is placed for decompression of small bowel obstruction, low, intermittent suction should begin early and continued as needed.

If gastropexy is used, the anchors can be safely cut one week later in our experience.

Tube clogging is typically from inadequately crushed pills or suboptimal flushing after use. The tube should be vigorously flushed with a syringe. If the above is unsuccessful, the tube should be exchanged. In the setting of a mature tract, if the tube is too obstructed to allow GW passage, it may be removed completely and the tract recanalized.

\section{PERCUTANEOUS RADIOLOGIC GASTROJEJU- NOSTOMY (PRGJ)}

PRGJ, so-called fluoroscopically guided transgastric jejunostomy is a procedure that involves placing a tube tip into the jejunum through a puncture of the stomach wall under fluoroscopic guidance. PRGJ can be done as an initial feeding tube placement (primary PRGJ) (Fig. 6) or can be made from conversion of prior gastrostomy to gastrojejunostomy (conversion PRGJ). Conversion may be performed any time after successful PRG placement if gastropexy is used, or if not, after the tract matures (usually in 1 to 3 weeks). In patients with a history or risk of GER or aspiration, PRGJ is considered, however, there is much controversy whether PRGJ could reduce incidence of GER or aspiration pneumonia compared with gastrostomy. ${ }^{14,15}$ Gastrojejunostomy tubes are longer and narrower than those placed in the stomach, and this could result in more frequent complications, such as tube blockage. $^{16}$

\section{1) Conversion PRGJ}

Conversion from gastrostomy to gastrojejunostomy can be considered when there are large gastric residuals or GER symptoms/aspiration pneumonia.

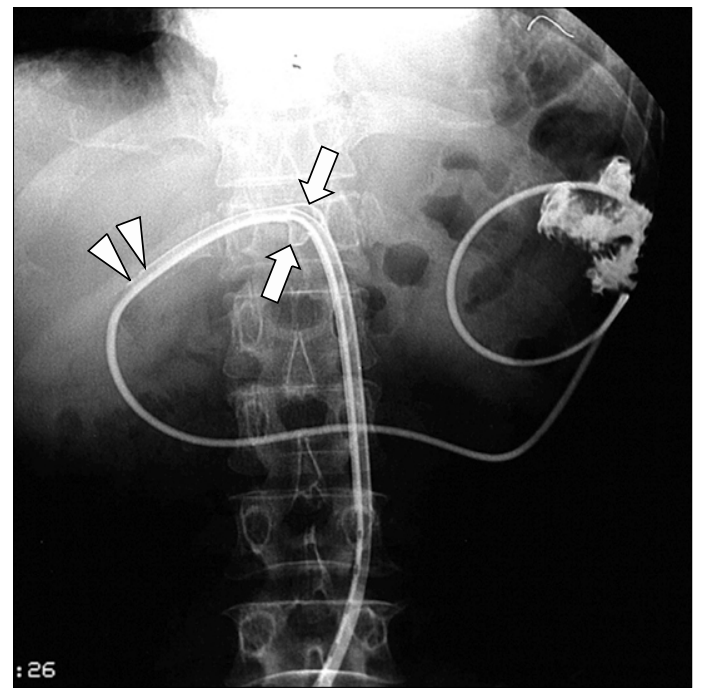

Fig. 6. Example of primary percutaneous radiologic gastrojejunostomy in a patient with recurrent aspiration pneumonia. A $16.5-\mathrm{Fr}, 80-\mathrm{cm}$, double-lumen gastrojejunostomy tube was then placed. A friction-lock Malecot retention device (arrows) is present to prevent inadvertent removal. The shorter lumen (arrowhead) within the stomach is for gastric suction. Contrast-agent investigation through the longer lumen reveals good opacification of the proximal jejunum without regurgitation into the stomach.

\section{2) Primary PRGJ}

Puncture direction should be toward the pylorus and positioning of the tube behind the ligament of Treitz is important to reduce the retrograde migration of the tube.

\section{PERCUTANEOUS RADIOLOGIC JEJUNOSTOMY (PRJ)}

It is indicated when prolonged enteral feeding is required in patients whose stomach is inaccessible for gastrostomy placement or those who have had a previous gastrectomy. ${ }^{16}$ Other less common indications include small bowel decompression for obstruction. Percutaneous puncture of the jejunum is more difficult than that of stomach because of the high mobility and easy collapsibility of the jejunum. The technical success of the PRJ placement ranges between $85 \%$ and $95 \% .{ }^{17-19}$ The reasons for failure include difficulty in puncturing the jejunum initially and subsequent dislodgement when inserting the anchor or tube. A 12 Fr or larger pigtail-retained gastrostomy tube is usually used. Particular care should be taken to avoid overlying large bowel loops, and decompression of the colon might need to be performed prior to jejunostomy insertion. ${ }^{16}$

Dilatation of jejunal loops is achieved by slow saline 
injection. Upon frontal and lateral fluoroscopy, the angiographic catheter tip is located in an air-filled proximal jejunal loop, as a target, in a portion that is sufficiently close to the anterior abdominal wall.

Distended jejunum is punctured under fluoroscopic and/or US guidance. The technique is similar to that of PRG tube placement. A 21-gauge Chiba needle can alternatively be used for puncture. ${ }^{17}$ After confirming the intraluminal positioning of the needle, a 0.018-in. GW is advanced as far as possible into the jejunum to allow insertion of 6 F. Neff catheter (Cook). A single Cope suture anchor is then deployed into the jejunum through the Neff catheter. Following serial dilation of the entry site, the tube is inserted.

\section{REFERENCES}

1. Laasch HU, Martin DF. Radiologic gastrostomy. Endoscopy 2007;39:247-255

2. Sinclair JJ, Scolapio JS, Stark ME, Hinder RA. Metastasis of head and neck carcinoma to the site of percutaneous endoscopic gastrostomy: case report and literature review. JPEN J Parenter Enteral Nutr 2001;25:282-285.

3. Shin $\mathrm{KH}$, Shin JH, Song HY, Yang ZQ, Kim JH, Kim KR. Primary and conversion percutaneous gastrojejunostomy under fluoroscopic guidance: 10 years of experience. Clin Imaging 2008;32:274-279.

4. Ho SG, Marchinkow LO, Legiehn GM, Munk PL, Lee MJ. Radiological percutaneous gastrostomy. Clin Radiol 2001; 56:902-910.

5. Kim JS, Park YW, Kim HK, et al. Is percutaneous endoscopic gastrostomy tube placement safe in patients with ventriculoperitoneal shunts? World J Gastroenterol 2009; 15:3148-3152.

6. Thornton FJ, Fotheringham T, Haslam PJ, McGrath FP, Keeling F, Lee MJ. Percutaneous radiologic gastrostomy with and without T-fastener gastropexy: a randomized comparison study. Cardiovasc Intervent Radiol 2002;25: 467-471.

7. Funaki B, Zaleski GX, Lorenz J, et al. Radiologic gastro- stomy placement: pigtail- versus mushroom-retained catheters. AJR Am J Roentgenol 2000;175:375-379.

8. Lyon SM, Haslam PJ, Duke DM, McGrath FP, Lee MJ. De novo placement of button gastrostomy catheters in an adult population: experience in 53 patients. J Vasc Interv Radiol 2003;14:1283-1289.

9. Kim JW, Song HY, Kim KR, Shin JH, Choi EK. The one-anchor technique of gastropexy for percutaneous radiologic gastrostomy: results of 248 consecutive procedures. J Vasc Interv Radiol 2008;19:1048-1053.

10. Brown AS, Mueller PR, Ferrucci JT Jr. Controlled percutaneous gastrostomy: nylon $\mathrm{T}$-fastener for fixation of the anterior gastric wall. Radiology 1986;158:543-545.

11. Wollman B, D'Agostino HB, Walus-Wigle JR, Easter DW, Beale A. Radiologic, endoscopic, and surgical gastrostomy: an institutional evaluation and meta-analysis of the literature. Radiology 1995;197:699-704.

12. Shin JH, Song HY, Kim TH, Kim KR, Choi KE, Kim JH. Percutaneous radiologic gastrostomy: a modified Chibaneedle puncture technique with single gastropexy. Abdom Imaging 2010;35:189-194.

13. Deutsch LS, Kannegieter L, Vanson DT, Miller DP, Brandon JC. Simplified percutaneous gastrostomy. Radiology 1992;184:181-183.

14. Hoffer EK, Cosgrove JM, Levin DQ, Herskowitz MM, Sclafani SJ. Radiologic gastrojejunostomy and percutaneous endoscopic gastrostomy: a prospective, randomized comparison. J Vasc Interv Radiol 1999;10:413-420.

15. Olson DL, Krubsack AJ, Stewart ET. Percutaneous enteral alimentation: gastrostomy versus gastrojejunostomy. Radiology 1993;187:105-108.

16. Given MF, Hanson JJ, Lee MJ. Interventional radiology techniques for provision of enteral feeding. Cardiovasc Intervent Radiol 2005;28:692-703.

17. Yang ZQ, Shin JH, Song HY, et al. Fluoroscopically guided percutaneous jejunostomy: outcomes in 25 consecutive patients. Clin Radiol 2007;62:1061-1065.

18. Cope C, Davis AG, Baum RA, Haskal ZJ, Soulen MC, Shlansky-Goldberg RD. Direct percutaneous jejunostomy: techniques and applications--ten years experience. Radiology 1998;209:747-754.

19. van Overhagen $\mathrm{H}$, Ludviksson MA, Lameris JS, et al. US and fluoroscopic-guided percutaneous jejunostomy: experience in 49 patients. J Vasc Interv Radiol 2000;11:101-106. 